\section{Tories out on cue}

Sir - In the issue of 30 September 1993, Landsberg, Dewynne and Please ${ }^{1}$ used a formula of mine ${ }^{2}$ to predict how long the Conservative government in Britain would continue its rule. My formula, based on the Copernican principle, stated that, if your location is not special, there is a 95 per cent chance that the future observed longevity of whatever you are observing now will be at least $1 / 39$ th as long as its past observed longevity but less than 39 times as long as its past observed longevity. Since the Conservative party had been in power for 14 years in 1993, Landsberg et al. estimated with 95 per cent confidence that it would remain in power for at least 4.3 more months but less than 546 more years.

The Conservative party lost power 3.6 years later, on 2 May 1997, in agreement with the prediction. This was a reasonable application of the formula, because it was prompted by the publication of my paper, whose publication date had no relation to British politics (unlike this letter). Indeed the claim of my paper is that its date of publication, 27 May 1993, is not special (except relative to itself, of course). The Copernican hypothesis can be tested: take an ensemble of things observable on my paper's date of publication and ask whether their future observability is correctly predicted by the formula 95 per cent of the time (see ref. 3 for some examples, from Broadway and offBroadway plays and musicals to world leaders' terms of office).

There have been other successful predictions. My paper used the above (95 per cent confidence) formula explicitly to predict that the human spaceflight programme would last at least 32/39ths of a year more — it has - and that Nature would continue publication for at least 3.15 more years - it has. The upper limits for these, 1,248 years and 4,800 years respectively, can be checked in the future. If the formula works well in all these applications, we may well take seriously my paper's ( 95 per cent confidence) prediction for the (200,000-year-old) human race as well: that its future longevity will be at least 5,100 years but less than 7.8 million years.

\section{J. Richard Gott III}

Department of Astrophysical Sciences,

Princeton University,

Princeton,

New Jersey 08544, USA

e-mail:jrg@astro.princeton.edu

1. Landsberg, P. T., Dewynne, J. N. \& Please, C. P. Nature 365, 384 (1993).

2. Gott, J. R. Nature 363, 315-319 (1993).

3. Gott, J. R. in Astronomical Society of the Pacific Conference Series (eds Trimble, V. \& Reisenegger, A.) 88, 140 (San Francisco, 1996).

\section{Stranglehold on science}

Sir - The attempt by P.-L. Chau and W. Y. Chau (Nature 386, 754; 1997) to refute Audrey Wells' observation of the dearth of individuality in Asian cultures as a consequence of Confucianism (Nature 386, 14; 1997) fails completely. They question whether it is "statistically significant to generalize from Western musical education in Korea and Japan in the past 30 years or so to arrive at a general conclusion about a complex 2,500-year-old philosophy that has deeply influenced the whole of East Asia". They then proceed to produce the same generalizations for which they so eagerly criticized Wells, by attempting to extrapolate present-day Asian culture from traditional Confucian philosophy.

Lacking any understanding of Joseph Needham and his writings, Chau and Chau question whether dualism or "paradoxes" have ever been connected to Taoism. Duality and paradox are intimately connected with Taoist philosophy, as any casual perusal of early Taoist texts would confirm. Furthermore, in Science and Civilization in China, Needham raises the point clearly and succinctly that, in fact, one of the possible reasons for the lack of a scientific revolution in China was the possibility that Confucianism imposed a 
collective stranglehold on Chinese intellectual advancement. In addition, one critical point that is studiously not addressed by Chau and Chau is Needham's observations regarding the culturally defined roles of science in Chinese society, in which practitioners of science (in the broadest sense) traditionally served the authoritarian needs of the state (royal court), particularly in close conjunction with well-defined élite classes that promulgated the systematic abuse of the predominantly submissive agrarian population.

To confound matters further, Chau and Chau seem not to understand that the concept of individualism has radically different meanings within the broader contexts of Asian and Western societies. They are also incorrect in stating that authoritarianism was unknown in the original writings of Confucian philosophers. On the contrary, Confucianism developed out of a society with a highly regimented social structure with little internal mobility; thus, virtue as a putatively beneficial quality was, and is, traditionally imposed from above in Chinese society. To the extent that moral courage existed in Chinese society, particularly during the Tang and Qing dynasties, it was a consequence of individual behaviour (and varied from person to person), not Confucianism. It was certainly not an idealistic consequence of well-travelled paths having been trodden in the search for greater meanings. Filial piety, therefore, is necessary for maintaining social harmony in Asian society. Confucian philosophers and scholars were hardly the only ones to have understood this point. Mao reformated this concept, and then used it to great ill-effect in his egotistical attempt to dominate Chinese society. Mao understood the Confucian Analects as well as any Chinese — probably more than most — and evidently to a much greater extent than the authors of the recent correspondence.

\section{Peter Cohen}

Department of Chemistry,

Columbia University,

MC 3150, Havemeyer Hall,

3000 Broadway,

New York, New York 10027, USA

e-mail:cohen@chem.columbia.edu

\section{You read it here first}

Sir - S. H. Friedman and J. O. M. Karlsson recently reported what they believed to be a novel paradigm (Nature 385, 480; 1997).

They presented a graph of the proportion of articles in the Medline database with the word 'novel' in the title or abstract, plotted as a function of the year of publication. They extrapolated, from the exponential rise in the use of the word, that by 2020 all scientific papers will claim novel findings. It is ironic that their observation is itself not novel.

Nature published correspondence in $1991(350,9 ; 1991)$ in which we noted the accelerating use of the word 'novel' and documented it with a graph of the frequency of use of 'novel' and two control words ('control' and 'unusual') in Medline database records. In addition, we recommended that authors reserve 'novel' for strikingly new discoveries, lest the word lose its impact.

We are sorry to see, from the updated data provided by Friedman and Karlsson, that our recommendation has had no discernible impact. It appears that the editors of Nature recognize the dire consequences of the novelty explosion and will provide regular updates of the situation until the message takes hold.

Robert W. Levis

Fred Hutchinson Cancer Research Center, Seattle,

Washington 98109, USA

Steven Henikoff

Fred Hutchinson Cancer Research Center, and Howard Hughes Medical Institute, Seattle,

Washington 98109, USA 